\title{
A COMPARISON OF MODELS OF A FOLDED PLATE
}

\author{
JOHN E. LAGNESE
}

\begin{abstract}
Two mathematical models of the junction region between two elastic plates will be compared. One of the models is due to H. Le Dret and was derived in the spirit of the Ciarlet-Destuynder method of asymptotic expansions. The other utilizes a classical Lagrangian formulation and is based on a certain type of geometric modeling. It is shown that in the case of a right-angle folded plate with "simple clamping," the two approaches give rise to one and the same model.
\end{abstract}

Key words: Distributed parameter modeling, junction region, folded plate, linked plates, plate network

Mathematics subject classification: $73 \mathrm{~K} 10,73 \mathrm{C} 35,35 \mathrm{Q} 99$

\section{INTRODUCTION}

The purpose of this paper is to compare mathematical models of the junction region between two elastic plates. More specifically, we wish to consider a model due to $H$. Le Dret that was derived in the spirit of the CiarletDestuynder method of asymptotic expansions, and another described by Lagnese, Leugering and Schmidt that utilizes the classical Lagrangian formulation and is based on a certain type of geometric modeling. The main point is that, at least in the case of a right-angle folded plate with "simple clamping," the two approaches give rise to one and the same model.

Work on modeling of a folded plate originated with Le Dret in a series of papers $[4],[5],[6],[7]$; see also the monograph [8]. A family of homogeneous, isotropic, linearly elastic three-dimensional bodies, consisting of two identical square plates of thickness $\varepsilon$ attached perpendicularly to each other, is considered. The Lamé parameters $\lambda_{\varepsilon}, \mu_{\varepsilon}$ of each of the bodies are assumed to satisfy $\left(\lambda_{\varepsilon}, \mu_{\varepsilon}\right)=\varepsilon^{-3}(\lambda, \mu)$. This assumption implies that the plates become progressively stiffer in certain directions as $\varepsilon \rightarrow 0$. The volume forces are also assumed to be scaled in a certain manner with respect to $\varepsilon$ with the precise scaling hypothesis depending on the assumed boundary conditions (i.e., either simple clamping - one of the two extreme edges is clamped and the other is free, or double clamping - both of the extreme edges are clamped). The idea is to show that under such scalings one may pass to the limit in the variational equations of the 3 - $d$ bodies as $\varepsilon \rightarrow 0$. The limit model consists of a pair of 2- $d$ equations of Kirchhoff type for the static equilibrium or dynamic motion, as appropriate, of the transverse displacements of the references surfaces of the two plates, the usual boundary conditions at the outer edges, and certain coupling conditions along the edge line formed by the intersection of the reference surfaces. The coupling conditions differ according to whether the configuration is simply or doubly clamped but in any case have reasonable geometric and mechanical interpretations. In the case of double clamping the coupling conditions are all local, but in the case of simple clamping some of the mechanical coupling conditions (balance laws) are nonlocal. Also, in the case of double clamping, continuity of the 
displacement vectors of the reference surfaces along the junction region may be lost in the limit.

Although Le Dret also considered more complicated configurations of linked plates (for example, two square plates meeting at an arbitrary angle), the approach described above, while very general and powerful, is suitable for modeling only relatively simple configurations. In contrast, the modeling approach introduced in [2] and [3] can be used to obtain models of the dynamics of quite complicated configurations in a fairly straightforward manner. In this approach, one begins by considering a two-dimensional network in $\mathbb{R}^{3}$, which is a subset of $\mathbb{R}^{3}$ formed by the union of a collection of relatively open, connected 2 - $\mathrm{d}$ sets $\mathcal{P}_{i}, i=1, \ldots, n$, each having a Lipschitz boundary consisting of a finite number of smooth curves, such that

(i) $\overline{\mathcal{P}}_{i} \cap \mathcal{P}_{j}=\emptyset, \quad \forall i \neq j$;

(ii) $\cup_{i=1}^{n} \overline{\mathcal{P}}_{i}$ is a connected set in $\mathbb{R}^{3}$;

(iii) $\overline{\mathcal{P}}_{i} \cap \overline{\mathcal{P}}_{j}$ is either empty or is a finite union of linear components, $\forall i \neq j$. We fix an orthonormal basis $\mathbf{a}_{1}^{i}, \mathbf{a}_{2}^{i}$ in $\mathcal{P}_{i}$ and set $\mathbf{a}_{3}^{i}=\mathbf{a}_{1}^{i} \times \mathbf{a}_{2}^{i}$. The set $\mathcal{P}_{i}$ is considered as the reference surface of a thin uniform plate whose reference configuration is

$$
\mathcal{B}_{i}=\left\{\mathcal{P}_{i}+\xi_{3} \mathbf{a}_{3}^{i}, 0<\xi_{3}<\varepsilon\right\},
$$

where $\varepsilon$ is a positive constant not depending on $i$. A junction of the network is a maximal, open line segment $J$ such that $\bar{J} \subset \overline{\mathcal{P}}_{i} \cap \overline{\mathcal{P}}_{j}$ for some $i \neq j$. Without loss of generality, it may be assumed that the junctions are mutually disjoint (see [3, Chapter VI, Section 2.1]). The index set $\mathcal{I}(J)$ of a junction $J$ is by definition

$$
\mathcal{I}(J)=\left\{i \in[1, \ldots, n] \mid J \subset \partial \mathcal{P}_{i}\right\} .
$$

Introduce Cartesian coordinates $\left(\xi_{1}, \xi_{2}\right)$ in $\mathcal{P}_{i}$ by writing

$$
\mathcal{P}_{i}=\left\{\mathbf{p}_{0}^{i}+\sum_{\alpha} \xi_{\alpha} \mathbf{a}_{\alpha}^{i}:=\mathbf{p}_{i}\left(\xi_{1}, \xi_{2}\right) \mid\left(\xi_{1}, \xi_{2}\right) \in \Omega_{i}\right\},
$$

where $\mathbf{p}_{0}^{i} \in \mathcal{P}_{i}$ denotes the origin of coordinates and $\Omega_{i} \subset \mathbb{R}^{2}$.

REMARK 1. Greek indices in a summation always take the values 1,2 , while Roman indices take values $1,2,3$ unless otherwise explicitly indicated.

Each plate $\mathcal{B}_{i}$ is assumed to satisfy the basic kinematic hypothesis of Reissner-Mindlin plate theory: the position vector to the displaced particle originally at $\mathbf{p}_{i}\left(\xi_{1}, \xi_{2}\right)+\xi_{3} \mathbf{a}_{3}^{i}$ is given by

$$
\mathbf{P}^{i}\left(\xi_{1}, \xi_{2}\right)+\xi_{3} \mathbf{A}_{3}^{i}\left(\xi_{1}, \xi_{2}\right), \quad\left(\xi_{1}, \xi_{2}\right) \in \Omega_{i}, 0<\xi_{3}<\varepsilon,
$$

where $\left|\mathbf{A}_{3}^{i}\right|=1$. Since we are interested in the dynamic deformation of the network, the quantities $\mathbf{P}^{i}, \mathbf{A}_{3}^{i}$ also depend on the time variable $t$, but explicit reference to $t$ will usually be suppressed. Set

$$
\begin{aligned}
\mathbf{P}^{i}-\mathbf{p}_{i}:=\mathbf{W}^{i} & =\sum_{j} W_{j}^{i} \mathbf{a}_{j}^{i}, \quad \mathbf{A}_{3}^{i}-\mathbf{a}_{3}^{i}:=\mathbf{U}^{i}=\sum_{j} U_{j}^{i} \mathbf{a}_{j}^{i}, \\
\mathbf{w}^{i} & =\sum_{\alpha} W_{\alpha}^{i} \mathbf{a}_{\alpha}^{i}, \quad \mathbf{u}^{i}=\sum_{\alpha} U_{\alpha}^{i} \mathbf{a}_{\alpha}^{i} .
\end{aligned}
$$

The quantity $\mathbf{W}^{i}$ is clearly the displacement vector of the reference surface $\mathcal{P}_{i}$, while $\mathbf{U}^{i}$ is associated with rotation of $\mathbf{a}_{3}^{i}$. Following [3], a junction $J$ is 
called rigid if the following conditions hold:

$$
\begin{gathered}
\mathbf{W}^{i}=\mathbf{W}^{j} \text { on } J, \quad \forall i, j \in \mathcal{I}(J) ; \\
\boldsymbol{\Phi}^{i}=\boldsymbol{\Phi}^{j} \text { on } J, \quad \forall i, j \in \mathcal{I}(J) ; \\
\mathbf{P}_{, 1}^{i} \cdot \mathbf{P}_{, 2}^{i}=0 \text { on } J, \quad \forall i \in \mathcal{I}(J) ; \\
J \text { undergoes rigid motion only. }
\end{gathered}
$$

The geometric meanings of (2) and (5) are obvious. Condition (4) means that the membrane strains vanish within the reference surface at the junction. In (3), $\Phi^{i}$ is called the rotation vector associated with the deformation of $\mathcal{P}_{i}$ and is a nonlinear constraint between the vectors $\mathbf{W}^{i}, \mathbf{U}^{i}$ and $\mathbf{W}^{j}, \mathbf{U}^{j}$ (see Appendix). It implies, in particular, that the angle between $\mathcal{P}_{i}$ and $\mathcal{P}_{j}$ remains invariant under the deformation for all $i, j \in \mathcal{I}(J)$. The linearization of (3) around the trivial equilibrium may be expressed as

$$
\begin{aligned}
-\left(\boldsymbol{\tau}^{i} \cdot \mathbf{u}^{i}\right) & \boldsymbol{\nu}^{i}+\left(\boldsymbol{\nu}^{i} \cdot \mathbf{u}^{i}\right) \boldsymbol{\tau}^{i}+\frac{1}{2}\left(\boldsymbol{\tau}^{i} \cdot \frac{\partial \mathbf{w}^{i}}{\partial \boldsymbol{\nu}^{i}}-\boldsymbol{\nu}^{i} \cdot \frac{\partial \mathbf{w}^{i}}{\partial \boldsymbol{\tau}^{i}}\right) \mathbf{a}_{3}^{i} \\
= & -\left(\boldsymbol{\tau}^{j} \cdot \mathbf{u}^{j}\right) \boldsymbol{\nu}^{j}+\left(\boldsymbol{\nu}^{j} \cdot \mathbf{u}^{j}\right) \boldsymbol{\tau}^{j}+\frac{1}{2}\left(\boldsymbol{\tau}^{j} \cdot \frac{\partial \mathbf{w}^{j}}{\partial \boldsymbol{\nu}^{j}}-\boldsymbol{\nu}^{j} \cdot \frac{\partial \mathbf{w}^{j}}{\partial \boldsymbol{\tau}^{j}}\right) \mathbf{a}_{3}^{j} .
\end{aligned}
$$

In this expression, $\boldsymbol{\nu}^{i}$ denotes the unit outward pointing normal to $\partial \mathcal{P}_{i}$ and $\boldsymbol{\tau}^{i}$ is a unit tangent vector oriented so that the triple $\boldsymbol{\nu}^{i}, \boldsymbol{\tau}^{i}, \mathbf{a}_{3}^{i}$ forms a right-handed orthonormal system. Equation (6) is the same as the pair of equations

$$
\begin{gathered}
\left(\boldsymbol{\nu}^{i} \cdot \mathbf{u}^{i}\right)=\sigma_{i j}\left(\boldsymbol{\nu}^{j} \cdot \mathbf{u}^{j}\right), \\
-\left(\boldsymbol{\tau}^{i} \cdot \mathbf{u}^{i}\right) \boldsymbol{\nu}^{i}+\frac{1}{2}\left(\boldsymbol{\tau}^{i} \cdot \frac{\partial \mathbf{w}^{i}}{\partial \boldsymbol{\nu}^{i}}-\boldsymbol{\nu}^{i} \cdot \frac{\partial \mathbf{w}^{i}}{\partial \boldsymbol{\tau}^{i}}\right) \mathbf{a}_{3}^{i} \\
=-\left(\boldsymbol{\tau}^{j} \cdot \mathbf{u}^{j}\right) \boldsymbol{\nu}^{j}+\frac{1}{2}\left(\boldsymbol{\tau}^{j} \cdot \frac{\partial \mathbf{w}^{j}}{\partial \boldsymbol{\nu}^{j}}-\boldsymbol{\nu}^{j} \cdot \frac{\partial \mathbf{w}^{j}}{\partial \boldsymbol{\tau}^{j}}\right) \mathbf{a}_{3}^{j}, \quad \forall i, j \in \mathcal{I}(J),
\end{gathered}
$$

where

$$
\sigma_{i j}(J)= \begin{cases}1 & \text { if } \boldsymbol{\tau}^{i}=\boldsymbol{\tau}^{j} \text { on } J \\ -1 & \text { if } \boldsymbol{\tau}^{i}=-\boldsymbol{\tau}^{j} \text { on } J\end{cases}
$$

The conditions (2) - (5) serve as "geometric" constraints on the deformation, along with any other geometric restrictions which may be introduced at the outer edges of the network (for example, if parts of the outer edges of one or more of the plates are clamped). Mechanical coupling conditions at the junction, together with the equations of motion and the mechanical boundary conditions, are then obtained by means of Hamilton's Principle, where the variation is taken with respect to deformations satisfying the imposed geometric constraints (rather, their linearizations), utilizing a Hamiltonian appropriate to a network of Reissner-Mindlin plates. Certain of the mechanical coupling conditions at the junction turn out to be nonlocal and have the flavor of those found by Le Dret in the situation of simple clamping described above. All of the mechanical conditions have plausible physical interpretations as balance laws. However, the two models (in the case of the configuration considered by Le Dret) are not directly comparable for two primary reasons. First, since the Le Dret approach is based on an assumption of elastic isotropy, it leads to a system of coupled Kirch hoff equations, while the model based on the kinematic assumption (1), the ad hoc constraints (2) 
- (5) and Hamilton's Principle involves a system of coupled Reissner-Mindlin equations. Second, in Le Dret's model, due to the assumed scaling of the Lamé parameters $\lambda_{\varepsilon}, \mu_{\varepsilon}$ with respect to $\varepsilon$, the membrane deformations in the limit model turn out to amount to rigid motions. Such motion implies (4) and (5), but is a much stronger constraint. Therefore, to effect a comparison between the two models, as a first step the constraints (4) and (5) are replaced by the more restrictive assumption

the membrane displacements of the reference surfaces are rigid.

Upon application of Hamilton's principle, this assumption, together with (2), (7), (8), leads to a certain model based on Reissner-Mindlin plate theory. It is then necessary to pass to the limit in this model as the shear moduli tend to infinity in order to enforce the Kirchhoff-Love hypothesis. When one carries out the limiting process, one finds that the resulting model is exactly the one found by Le Dret.

The plan of the remainder of this paper is as follows. In the next section, Hamilton's Principle is used to derive equations of motion and mechanical junction and boundary conditions for a 2-d network consisting of $n$ planar regions which share a common joint $J$, under the kinematic assumption (1) and the geometric constraints $(2),(7),(8)$ and (9). In order to compare against the Le Dret model, it is assumed that all of the reference surfaces are unit squares and that exactly one or them is clamped at an extreme edge (the edge not adjacent to the junction) while all of the remaining outer edges are free. However, we allow the plates to have differing material properties. The limit model as the shear moduli tend to infinity is derived in section 3 . It is observed that when the limit model is specialized to the case of two identical square plates which are orthogonal to each other, what is obtained is exactly the model derived by Le Dret.

REMARK 2. The junction conditions found by Le Dret [5] in the case of a doubly clamped pair of orthogonal plates do not correspond to a rigid junction as defined above nor to any of the types of junctions defined in [3, Chapter VII, Section 3]. In all cases, the approach of [3] enforces the continuity condition (2) while, as noted above, such is not the case in the model obtained in [5], where continuity of only the tangential components of displacements at the junction is assured.

\section{Derivation OF THE MODEL}

Let $\mathbf{i}, \mathbf{j}, \mathbf{k}$ denote the standard basis for $\mathbb{R}^{3}$ and $\left(x_{1}, x_{2}, x_{3}\right)$ denote the Cartesian coordinates of a point in $\mathbb{R}^{3}$ with respect to that basis. We consider a 2-d network in $\mathbb{R}^{3}$ of consisting of planar regions $\mathcal{P}_{i}, i=1, \ldots, n$, such that

(i) $\mathcal{P}_{i}$ is a unit square;

(ii) $\overline{\mathcal{P}}_{i} \cap \overline{\mathcal{P}}_{j}=\left\{\left(0,0, x_{3}\right)|| x_{3} \mid \leq 1 / 2\right\}:=J$, for all $i \neq j$.

If $n=2$ it is further assumed that $\mathcal{P}_{1}$ and $\mathcal{P}_{2}$ are not coplanar. Let $\boldsymbol{\nu}_{J}^{i}$ denote the exterior unit normal to $\partial \mathcal{P}_{i}$ along $J$ and set $\tau=\mathrm{k}$. One may write

$$
\mathcal{P}_{i}=-\xi_{1} \nu_{J}^{i}+\left(\xi_{2}-1 / 2\right) \tau, \quad 0<\xi_{i}<1 .
$$

This conforms to the notation of section 1 if we set $\mathbf{a}_{1}^{i}=-\boldsymbol{\nu}_{J}^{i}, \mathbf{a}_{2}^{i}=\boldsymbol{\tau}, \mathbf{a}_{3}^{i}=$ $\mathbf{a}_{1}^{i} \times \mathbf{a}_{2}^{i}$, and $\Omega_{i}=\left\{\left(\xi_{1}, \xi_{2}\right) \mid 0<\xi_{1}<1,-1 / 2<\xi_{2}<1 / 2\right\}, i=1, \ldots, n$. As 
there, the components of the displacement vector $\mathbf{W}^{i}$ and the vector $\mathbf{U}^{i}$ with respect to the $\mathbf{a}_{j}^{i}$ basis are denoted by $W_{j}^{i}$ and $U_{j}^{i}$, respectively, $j=1,2,3$.

One plate of the network is assumed to be clamped at its extreme edge; we assume it to be $\mathcal{P}_{1}$, so that

$$
\mathbf{W}^{1}\left(1, \xi_{2}\right)=0, \quad \mathbf{u}^{1}\left(1, \xi_{2}\right)=0, \quad 0<\xi_{2}<1 .
$$

Further, because of the way the bases were chosen, the continuity condition (2) takes the form

$$
\begin{gathered}
W_{1}^{i}\left(0, \xi_{2}\right) \mathbf{a}_{1}^{i}+W_{3}^{i}\left(0, \xi_{2}\right) \mathbf{a}_{3}^{i}=W_{1}^{j}\left(0, \xi_{2}\right) \mathbf{a}_{1}^{j}+W_{3}^{j}\left(0, \xi_{2}\right) \mathbf{a}_{3}^{j}, \\
W_{2}^{i}\left(0, \xi_{2}\right)=W_{2}^{j}\left(0, \xi_{2}\right), \quad 0<\xi_{2}<1, \quad i, j=1, \ldots, n,
\end{gathered}
$$

while $(7)$ is

$$
U_{1}^{i}\left(0, \xi_{2}\right)=U_{1}^{j}\left(0, \xi_{2}\right), \quad 0<\xi_{2}<1, \quad i, j=1, \ldots, n,
$$

and $(8)$ is

$$
\begin{aligned}
{\left[-U_{2}^{i} \mathbf{a}_{1}^{i}+\frac{1}{2}\left(\mathbf{a}_{2}^{i} \cdot \frac{\partial \mathbf{w}^{i}}{\partial \xi_{1}}-\mathbf{a}_{1}^{i} \cdot \frac{\partial \mathbf{w}^{i}}{\partial \xi_{2}}\right) \mathbf{a}_{3}^{i}\right] } & \left(0, \xi_{2}\right) \\
= & {\left[-U_{2}^{j} \mathbf{a}_{1}^{j}+\frac{1}{2}\left(\mathbf{a}_{2}^{j} \cdot \frac{\partial \mathbf{w}^{j}}{\partial \xi_{1}}-\mathbf{a}_{1}^{j} \cdot \frac{\partial \mathbf{w}^{j}}{\partial \xi_{2}}\right) \mathbf{a}_{3}^{j}\right]\left(0, \xi_{2}\right), } \\
& 0<\xi_{2}<1, i, j=1, \ldots, n .
\end{aligned}
$$

Consider the constraint (9). It follows from it that the components of membrane displacement $\mathbf{w}^{i}\left(\xi_{1}, \xi_{2}\right)$ with respect to $\mathbf{a}_{1}^{i}, \mathbf{a}_{2}^{i}$ are given by

$$
\left(\begin{array}{cc}
\cos \vartheta_{i}-1 & \sin \vartheta_{i} \\
-\sin \vartheta_{i} & \cos \vartheta_{i}-1
\end{array}\right)\left(\begin{array}{c}
\xi_{1} \\
\xi_{2}-1 / 2
\end{array}\right)+\left(\begin{array}{l}
b_{i}^{1} \\
b_{i}^{2}
\end{array}\right)
$$

for some rotation angle $\vartheta_{i}$ and translation vector $b_{i}^{1} \mathbf{a}_{1}^{i}+b_{i}^{2} \mathbf{a}_{2}^{i}$. Since only linear theory will be considered, (14) is replaced by its linearization

$$
\mathbf{w}^{i}\left(\xi_{1}, \xi_{2}\right)=\left[\vartheta_{i}\left(\xi_{2}-1 / 2\right)+b_{i}^{1}\right] \mathbf{a}_{1}^{i}+\left(-\vartheta_{i} \xi_{1}+b_{i}^{2}\right) \mathbf{a}_{2}^{i} .
$$

From (10), (11), (13) and (15) we deduce that

$$
\vartheta_{1}=b_{1}^{1}=0, \quad b_{i}^{2}=0, \quad i=1, \ldots, n,
$$

that

$$
W_{3}^{1}\left(0, \xi_{2}\right) \mathbf{a}_{3}^{1}=\left[\vartheta_{i}\left(\xi_{2}-1 / 2\right)+b_{i}\right] \mathbf{a}_{1}^{i}+W_{3}^{i}\left(0, \xi_{2}\right) \mathbf{a}_{3}^{i}, \quad i=2, \ldots, n,
$$

where $b_{i}:=b_{i}^{1}$, and that

$$
U_{2}^{1}\left(0, \xi_{2}\right) \mathbf{a}_{1}^{1}=U_{2}^{i}\left(0, \xi_{2}\right) \mathbf{a}_{1}^{i}+\vartheta_{i} \mathbf{a}_{3}^{i}, \quad i=2, \ldots, n .
$$

The relation (17) is equivalent to

$$
\begin{gathered}
\vartheta_{i}\left(\xi_{2}-1 / 2\right)+b_{i}=\left(\mathbf{a}_{3}^{1} \cdot \mathbf{a}_{1}^{i}\right) W_{3}^{1}\left(0, \xi_{2}\right), \\
W_{3}^{i}\left(0, \xi_{2}\right)=\left(\mathbf{a}_{3}^{1} \cdot \mathbf{a}_{3}^{i}\right) W_{3}^{1}\left(0, \xi_{2}\right), \quad i=2, \ldots, n .
\end{gathered}
$$

If $\mathcal{P}_{1}$ is coplanar with $\mathcal{P}_{i}$ for some index $i \geq 2$, then $\mathbf{a}_{3}^{1} \cdot \mathbf{a}_{1}^{i}=0$ and $\mathbf{a}_{3}^{1} \cdot \mathbf{a}_{3}^{i}=-1$ so that $\vartheta_{i}=b_{i}=0$ and $W_{3}^{i}\left(0, \xi_{2}\right)=-W_{3}^{1}\left(0, \xi_{2}\right)$ for that index. In order to simplify the presentation, it is assumed that $\mathcal{P}_{1}$ and $\mathcal{P}_{i}$ are not coplanar for any $i=2, \ldots, n$. (This assumption does not change what follows in any essential way.) Then we may conclude from (19) that

$$
\vartheta_{i}=k_{i} \vartheta, \quad b_{i}=k_{i} b, \quad i=2, \ldots, n,
$$


where $\vartheta:=\vartheta_{2}, b:=b_{2}$ and

$$
k_{i}=\frac{\mathbf{a}_{3}^{1} \cdot \mathbf{a}_{1}^{i}}{\mathbf{a}_{3}^{1} \cdot \mathbf{a}_{1}^{2}}= \pm \frac{\sin \alpha_{i}}{\sin \alpha_{2}}
$$

with $\alpha_{i}$ denoting the angle between $\mathcal{P}_{1}$ and $\mathcal{P}_{i}, 0<\alpha_{i}<\pi$. Furthermore, we obtain

$$
W_{3}^{i}\left(0, \xi_{2}\right)=k_{i}^{\prime}\left[\vartheta\left(\xi_{2}-1 / 2\right)+b\right], \quad i=1, \ldots, n,
$$

where

$$
k_{i}^{\prime}=\frac{\mathbf{a}_{3}^{1} \cdot \mathbf{a}_{3}^{i}}{\mathbf{a}_{3}^{1} \cdot \mathbf{a}_{1}^{2}}=\frac{\cos \alpha_{i}}{ \pm \sin \alpha_{2}} .
$$

Similarly, using $\mathbf{a}_{1}^{1} \cdot \mathbf{a}_{3}^{i}=-\mathbf{a}_{3}^{1} \cdot \mathbf{a}_{1}^{i}$ and $\mathbf{a}_{1}^{1} \cdot \mathbf{a}_{1}^{i}=\mathbf{a}_{3}^{1} \cdot \mathbf{a}_{3}^{i}$, one finds that (18) is equivalent to

$$
U_{2}^{i}\left(0, \xi_{2}\right)=-k_{i}^{\prime} \vartheta, \quad i=1, \ldots, n .
$$

The constraints $(12),(13)$ therefore reduce to

$$
U_{1}^{i}\left(0, \xi_{2}\right)=U_{1}^{j}\left(0, \xi_{2}\right), \quad U_{2}^{i}\left(0, \xi_{2}\right)=-k_{i}^{\prime} \vartheta, \quad i, j=1, \ldots, n .
$$

The state variables of the problem are $W_{3}^{i}, U_{1}^{i}, U_{2}^{i}, i=1, \ldots, n$; and $\vartheta, b$. These are constrained by $(20),(21)$ and

$$
W_{3}^{1}\left(1, \xi_{2}\right)=U_{1}^{1}\left(1, \xi_{2}\right)=U_{2}^{1}\left(1, \xi_{2}\right)=0 .
$$

Remark 3 . In the case where $n=2$ and $\mathcal{P}_{1}$ is orthogonal to $\mathcal{P}_{2},(20)$ reduces to

$$
W_{3}^{1}\left(0, \xi_{2}\right)= \pm\left[\vartheta\left(\xi_{2}-1 / 2\right)+b\right], \quad W_{3}^{2}\left(0, \xi_{2}\right)=0,
$$

and (21) becomes

$$
U_{1}^{1}\left(0, \xi_{2}\right)=U_{1}^{2}\left(0, \xi_{2}\right), \quad U_{2}^{1}\left(0, \xi_{2}\right)=\mp \vartheta, \quad U_{2}^{2}\left(0, \xi_{2}\right)=0 .
$$

We now utilize Hamilton's principle

$$
\delta \int_{0}^{T}[K(t)-U(t)+W(t)] d t=0
$$

to derive the equations of motion and the mechanical junction conditions. The Lagrangian employed is based on energy functionals appropriate to Reissner-Mindlin plate theory. Let $\rho_{i}$ denote the mass density per unit of reference volume of the $i$-th plate, and set $m_{i}=\varepsilon \rho_{i}, I_{\rho_{i}}=\left(\varepsilon^{3} / 12\right) \rho_{i}$. Let $\omega=[0,1] \times[0,1], d \omega=d \xi_{1} d \xi_{2}$. The kinetic energy $K(t)$ is defined by

$$
\begin{aligned}
2 K(t)= & \sum_{i=1}^{n} \int_{\omega}\left(m_{i}\left|\dot{\mathbf{W}}^{i}\right|^{2}+I_{\rho_{i}}\left|\dot{\mathbf{u}}^{i}\right|^{2}\right) d \omega \\
= & \sum_{i=1}^{n} \int_{\omega}\left(m_{i}\left|\dot{W}_{3}^{i}\right|^{2}+I_{\rho_{i}}\left|\dot{\mathbf{u}}^{i}\right|^{2}\right) d \omega \\
& \quad+\sum_{i=2}^{n} m_{i} k_{i}^{2} \int_{\omega}\left[\left|\dot{\vartheta}\left(\xi_{2}-1 / 2\right)+\dot{b}\right|^{2}+\xi_{1}^{2} \dot{\vartheta}^{2}\right] d \omega \\
= & \sum_{i=1}^{n} \int_{\omega}\left(m_{i}\left|\dot{W}_{3}^{i}\right|^{2}+I_{\rho_{i}}\left|\dot{\mathbf{u}}^{i}\right|^{2}\right) d \omega+M\left(\frac{5}{12} \dot{\vartheta}^{2}+\dot{b}^{2}\right)
\end{aligned}
$$

where $M=\sum_{i=2}^{n} m_{i} k_{i}^{2}$ and ${ }^{\circ}=\partial / \partial t$. 
Let $\mathcal{S}$ denote the set of all $2 \times 2$ symmetric matrices. For any $e \in \mathcal{S}$ define $C_{i}[e] \in \mathcal{S}$ by

$$
C_{i}[e]=\frac{\mu_{i} \varepsilon^{3}}{6}\left[e+\frac{\lambda_{i}}{2 \mu_{i}+\lambda_{i}} \operatorname{trace}(e) \mathbf{i d}\right],
$$

where id is the identity in $\mathcal{S}$ and $\lambda_{i}, \mu_{i}$ denote material parameters associated with the $i$-th plate. The strain energy functional $U(t)$ is given by

$$
2 U(t)=\sum_{i=1}^{n} \int_{\omega}\left\{C_{i}\left[e\left(\mathbf{u}^{i}\right)\right]: e\left(\mathbf{u}^{i}\right)+G_{i} \varepsilon\left|\mathbf{u}^{i}+\nabla W_{3}^{i}\right|^{2}\right\} d \omega
$$

where

$$
e\left(\mathbf{u}^{i}\right)=\frac{1}{2}\left(U_{\alpha, \beta}^{i}+U_{\beta, \alpha}^{i}\right)
$$

and where $A: B=\operatorname{trace} A^{T} B, \nabla W_{3}^{i}=\sum_{\alpha} W_{3, \alpha}^{i} \mathbf{a}_{\alpha}^{i}$, and $G_{i}$ is the shear modulus of the $i$ th plate.

Assume that the plates are subject to distributed loadings $\mathbf{F}^{i}=\sum_{j} F_{j}^{i} \mathbf{a}_{j}^{i}$ only. In order to be consistent with (9) it is reasonable to assume that the membrane forces in $\mathcal{P}_{1}$ vanish, i.e., $F_{1}^{1}=F_{2}^{1}=0$. The work done on the plate network by these forces is

$$
W(t)=\sum_{i=1}^{n} \int_{\omega}\left(\mathbf{f}^{i} \cdot \mathbf{W}^{i}+\mathbf{M}^{i} \cdot \mathbf{u}^{i}\right) d \omega
$$

where

$$
\begin{gathered}
\mathbf{f}^{i}\left(\xi_{1}, \xi_{2}\right)=\int_{0}^{\varepsilon} \mathbf{F}^{i}\left(\xi_{1}, \xi_{2}, \xi_{3}\right) d \xi_{3} \\
\mathbf{M}^{i}\left(\xi_{1}, \xi_{2}\right)=\int_{0}^{\varepsilon}\left(\xi_{3}-\varepsilon / 2\right) \sum_{\alpha} F_{\alpha}^{i}\left(\xi_{1}, \xi_{2}, \xi_{3}\right) \mathbf{a}_{\alpha}^{i} d \xi_{3} .
\end{gathered}
$$

Write $\mathbf{f}^{i}=\sum_{j} f_{j}^{i} \mathbf{a}_{j}^{i}$. The vanishing of membrane forces in $\mathcal{P}_{1}$ implies that $\mathbf{M}^{1}=0$ and that $\mathbf{f}^{1}=f_{3}^{1} \mathbf{a}_{3}^{1}$. Then

$$
\begin{aligned}
W(t)= & \sum_{i=1}^{n} \int_{\omega} f_{3}^{i} W_{3}^{i} d \omega \\
& +\sum_{i=2}^{n} \int_{\omega}\left\{\mathbf{M}^{i} \cdot \mathbf{u}^{i}+k_{i}\left\{f_{1}^{i}\left[\vartheta\left(\xi_{2}-1 / 2\right)+b\right]-f_{2}^{i} \vartheta \xi_{1}\right\}\right\} d \omega .
\end{aligned}
$$

We may now calculate (25), where the variation is taken with respect to test functions satisfying (20) - (22) for $0<t<T$ and vanishing at $t=0$ and $t=T$. In this calculation, use is made of the following Green's formula (see Ciarlet [1])

$$
\begin{aligned}
\int_{\omega} C_{i}\left[e\left(\mathbf{u}^{i}\right)\right]: e\left(\hat{\mathbf{u}}^{i}\right) d \omega=-\int_{\omega} \hat{\mathbf{u}}^{i} \cdot \operatorname{div} C_{i}\left[e\left(\mathbf{u}^{i}\right)\right] d \omega & \\
& +\int_{\partial \omega} \hat{\mathbf{u}}^{i} \cdot\left(C_{i}\left[e\left(\mathbf{u}^{i}\right)\right] \nu^{i}\right) d s
\end{aligned}
$$

where $\boldsymbol{\nu}=\left(\nu_{1}, \nu_{2}\right)$ is the unit outer normal to $\partial \omega, \boldsymbol{\nu}^{i}=\sum_{\alpha} \nu_{\alpha} \mathbf{a}_{\alpha}^{i}$, and

$$
\operatorname{div} C_{i}\left[e\left(\mathbf{u}^{i}\right)\right]=\sum_{\alpha, \beta} \frac{\partial}{\partial \xi_{\alpha}} C_{i}^{\alpha \beta}\left[e\left(\mathbf{u}^{i}\right)\right] \mathbf{a}_{\beta}^{i}, \quad C_{i}\left[e\left(\mathbf{u}^{i}\right)\right] \boldsymbol{\nu}^{i}=\sum_{\alpha, \beta} C_{i}^{\alpha \beta}\left[e\left(\mathbf{u}^{i}\right)\right] \nu_{\alpha} \mathbf{a}_{\beta}^{i} .
$$


One obtains the following variational equation, which must hold for all sufficiently smooth test functions $\hat{W}_{3}^{i}, \hat{\mathbf{u}}^{i}$ and all real numbers $\hat{\theta}, \hat{b}$ which satisfy $(20)-(22)$ :

$$
\begin{aligned}
0=- & M\left(\frac{5}{12} \ddot{\vartheta} \hat{\vartheta}+\ddot{b} \hat{b}\right)+\sum_{i=1}^{n} \int_{\omega}\left\{-m_{i} \ddot{W}_{3}^{i} \hat{W}_{3}^{i}-I_{\rho_{i}} \ddot{\mathbf{u}}^{i} \cdot \hat{\mathbf{u}}^{i}+\hat{\mathbf{u}}^{i} \cdot \operatorname{div} C_{i}\left[e\left(\mathbf{u}^{i}\right)\right]\right. \\
& \left.-G_{i} \varepsilon \hat{\mathbf{u}}^{i} \cdot\left(\mathbf{u}^{i}+\nabla W_{3}^{i}\right)+G_{i} \varepsilon \hat{W}_{3}^{i} \operatorname{div}\left(\mathbf{u}^{i}+\nabla W_{3}^{i}\right)\right\} d \omega \\
& -\sum_{i=1}^{n} \int_{\partial \omega}\left\{\hat{\mathbf{u}}^{i} \cdot\left(C_{i}\left[e\left(\mathbf{u}^{i}\right)\right] \boldsymbol{\nu}^{i}\right)+G_{i} \varepsilon \hat{W}_{3}^{i} \nu^{i} \cdot\left(\mathbf{u}^{i}+\nabla W_{3}^{i}\right)\right\} d s \\
+ & \sum_{i=2}^{n} \int_{\omega}\left\{\mathbf{M}^{i} \cdot \hat{\mathbf{u}}^{i}+k_{i}\left\{\left[f_{1}^{i}\left(\xi_{2}-1 / 2\right)-f_{2}^{i} \xi_{1}\right] \hat{\vartheta}+f_{1}^{i} \hat{b}\right\}\right\} d \omega \\
& +\sum_{i=1}^{n} \int_{\omega} f_{3}^{i} \hat{W}_{3}^{i} d \omega, \quad 0<t<T
\end{aligned}
$$

Set

$$
\gamma=\left\{\left(0, \xi_{2}\right) \mid 0<\xi_{2}<1\right\}, \quad \gamma_{1}=\left\{\left(1, \xi_{2}\right) \mid 0<\xi_{2}<1\right\} .
$$

From (27) one obtains the equations of motion

$$
\left\{\begin{array}{l}
m_{i} \ddot{W}_{3}^{i}-G_{i} \varepsilon \operatorname{div}\left(\mathbf{u}^{i}+\nabla W_{3}^{i}\right)=f_{3}^{i} \\
I_{\rho_{i}} \ddot{\mathbf{u}}^{i}-\operatorname{div} C_{i}\left[\epsilon\left(\mathbf{u}^{i}\right)\right]+G_{i} \varepsilon\left(\mathbf{u}^{i}+\nabla W_{3}^{i}\right)=\mathbf{M}^{i}
\end{array}\right.
$$

where $\mathbf{M}^{1}=0$, the boundary conditions

$$
\left\{\begin{array}{l}
C_{i}\left[e\left(\mathbf{u}^{i}\right)\right] \boldsymbol{\nu}^{i}=0, \quad \boldsymbol{\nu}^{i} \cdot\left(\mathbf{u}^{i}+\nabla W_{3}^{i}\right)=0 \text { on } \partial \omega \backslash \gamma, i=2, \ldots, n, \\
C_{1}\left[e\left(\mathbf{u}^{1}\right)\right] \boldsymbol{\nu}^{1}=0, \quad \boldsymbol{\nu}^{1} \cdot\left(\mathbf{u}^{1}+\nabla W_{3}^{1}\right)=0 \text { on } \partial \omega \backslash\left(\gamma \cup \gamma_{1}\right),
\end{array}\right.
$$

together with the variational junction condition

$$
\begin{array}{r}
M\left(\frac{5}{12} \ddot{\vartheta} \hat{\vartheta}+\ddot{b} \hat{b}\right)=-\sum_{i=1}^{n} \int_{\gamma}\left\{\hat{\mathbf{u}}^{i} \cdot\left(C_{i}\left[e\left(\mathbf{u}^{i}\right)\right] \boldsymbol{\nu}^{i}\right)+G_{i} \varepsilon \hat{W}_{3}^{i} \boldsymbol{\nu}^{i} \cdot\left(\mathbf{u}^{i}+\nabla W_{3}^{i}\right)\right\} d \xi_{2} \\
+\sum_{i=2}^{n} k_{i} \int_{\omega}\left\{\left[f_{1}^{i}\left(\xi_{2}-1 / 2\right)-f_{2}^{i} \xi_{1}\right] \hat{\vartheta}+f_{1}^{i} \hat{b}\right\} d \omega
\end{array}
$$

It remains to interpret $(30)$. On $\gamma,\left(\nu_{1}, \nu_{2}\right)=(-1,0)$, hence

$$
\begin{gathered}
C_{i}\left[e\left(\mathbf{u}^{i}\right)\right] \boldsymbol{\nu}^{i}=-\sum_{\beta} C_{i}^{1, \beta}\left[e\left(\mathbf{u}^{i}\right)\right] \mathbf{a}_{\beta}^{i} \\
=-\frac{\varepsilon^{3}}{12}\left[\frac{4 \mu_{i}\left(\mu_{i}+\lambda_{i}\right)}{2 \mu_{i}+\lambda_{i}} U_{1,1}^{i} \mathbf{a}_{1}^{i}+\mu_{i}\left(U_{1,2}^{1}+U_{2,1}^{i}\right) \mathbf{a}_{2}^{i}\right], \\
\boldsymbol{\nu}^{i} \cdot\left(\mathbf{u}^{i}+\nabla W_{3}^{i}\right)=-U_{1}^{i}-W_{3,1}^{i}=-U_{1}^{1}-W_{3,1}^{i},
\end{gathered}
$$

where we have used (20) and (21). Further, on $\gamma$,

$$
\begin{gathered}
\hat{\mathbf{u}}^{i} \cdot\left(C_{i}\left[e\left(\mathbf{u}^{i}\right)\right] \boldsymbol{\nu}^{i}\right)=\frac{\varepsilon^{3}}{12}\left[-\frac{4 \mu_{i}\left(\mu_{i}+\lambda_{i}\right)}{2 \mu_{i}+\lambda_{i}} U_{1,1}^{i} \hat{U}_{1}^{1}+k_{i}^{\prime} \mu_{i}\left(U_{1,2}^{1}+U_{2,1}^{i}\right) \hat{\vartheta}\right], \\
\hat{W}_{3}^{i} \boldsymbol{\nu}^{i} \cdot\left(\mathbf{u}^{i}+\nabla W_{3}^{i}\right)=-k_{i}^{\prime}\left(U_{1}^{1}+W_{3,1}^{i}\right)\left[\hat{\vartheta}\left(\xi_{2}-1 / 2\right)+\hat{b}\right] .
\end{gathered}
$$


Therefore, (30) is

$$
\begin{aligned}
& M\left(\frac{5}{12} \ddot{\vartheta} \hat{\vartheta}+\ddot{b} \hat{b}\right)=\sum_{i=1}^{n} \varepsilon \int_{\gamma}\left\{\frac { \varepsilon ^ { 2 } } { 1 2 } \left[\frac{4 \mu_{i}\left(\mu_{i}+\lambda_{i}\right)}{2 \mu_{i}+\lambda_{i}} U_{1,1}^{i} \hat{U}_{1}^{1}\right.\right. \\
&\left.\left.-k_{i}^{\prime} \mu_{i}\left(U_{1,2}^{1}+U_{2,1}^{i}\right) \hat{\vartheta}\right]+k_{i}^{\prime} G_{i}\left(U_{1}^{1}+W_{3,1}^{i}\right)\left[\hat{\vartheta}\left(\xi_{2}-1 / 2\right)+\hat{b}\right]\right\} d \xi_{2} \\
&+\sum_{i=2}^{n} k_{i} \int_{\omega}\left\{\left[f_{1}^{i}\left(\xi_{2}-1 / 2\right)-f_{2}^{i} \xi_{1}\right] \hat{\vartheta}+f_{1}^{i} \hat{b}\right\} d \omega .
\end{aligned}
$$

It follows from (31) that the mechanical junction conditions are

$$
\begin{gathered}
\sum_{i=1}^{n} \frac{\mu_{i}\left(\mu_{i}+\lambda_{i}\right)}{2 \mu_{i}+\lambda_{i}} U_{1,1}^{i}\left(0, \xi_{2}\right)=0 \\
M \frac{5}{12} \ddot{\vartheta}=\sum_{i=1}^{n} k_{i}^{\prime} \varepsilon \int_{\nu}\left[-\frac{\mu_{i} \varepsilon^{2}}{12}\left(U_{1,2}^{1}+U_{2,1}^{i}\right)+G_{i}\left(U_{1}^{1}+W_{3,1}^{i}\right)\left(\xi_{2}-1 / 2\right)\right] d \xi_{2} \\
+\sum_{i=2}^{n} k_{i} \int_{\omega}\left[f_{1}^{i}\left(\xi_{2}-1 / 2\right)-f_{2}^{i} \xi_{1}\right] d \omega \\
M \ddot{b}=\sum_{i=1}^{n} k_{i}^{\prime} G_{i} \varepsilon \int_{\gamma}\left(U_{1}^{1}+W_{3,1}^{i}\right) d \xi_{2}+\sum_{i=2}^{n} k_{i} \int_{\omega} f_{1}^{i} d \omega
\end{gathered}
$$

REMARK 4 . In the special case where $n=2$ and $\mathcal{P}_{1}$ is orthogonal to $\mathcal{P}_{2}$, one has $k_{1}^{\prime}= \pm 1, k_{2}^{\prime}=0, k_{2}=1$. If also the material parameters of the two plates are identical, the last three equations reduce to

$$
\begin{gathered}
U_{1,1}^{1}\left(0, \xi_{2}\right)=-U_{1,1}^{2}\left(0, \xi_{2}\right) \\
\frac{5}{12} \varepsilon \rho_{2} \ddot{\vartheta}=\varepsilon k_{1}^{\prime} \int_{\gamma}\left[-\frac{\mu \varepsilon^{2}}{12}\left(U_{1,2}^{1}+U_{2,1}^{1}\right)+G\left(U_{1}^{1}+W_{3,1}^{1}\right)\left(\xi_{2}-1 / 2\right)\right] d \xi_{2} \\
+\int_{\omega}\left[f_{1}^{2}\left(\xi_{2}-1 / 2\right)-f_{2}^{2} \xi_{1}\right] d \omega \\
\varepsilon \rho_{2} \ddot{b}=k_{1}^{\prime} G \varepsilon \int_{\gamma}\left(U_{1}^{1}+W_{3,1}^{1}\right) d \xi_{2}+\int_{\omega} f_{1}^{2} d \omega .
\end{gathered}
$$

The junction conditions (20), (21), (35) - (37) may be considered as analogous to those found by Le Dret for a simply clamped folded plate, but within the context of Reissner-Mindlin plate theory rather than KirchhoffLove plate theory.

\section{THE LIMIT MODEL}

In order to obtain a model appropriate for comparison with the model derived by Le Dret, we consider the limit of the model derived above as the shear moduli $G_{i} \rightarrow \infty$. First, it is necessary to show the above model is well-posed in an appropriate function space. To that end, we set, for scalar functions $\varphi_{1}^{i}, \varphi_{2}^{i}, \zeta^{i}$ defined on $\omega$ and real numbers $\vartheta, b$,

$$
\mathbf{R}^{i}=\sum_{\alpha} \varphi_{\alpha}^{i} \mathbf{a}_{\alpha}^{i}+\zeta^{i} \mathbf{a}_{3}^{i}:=\varphi^{i}+\zeta^{i} \mathbf{a}_{3}^{i}, \quad \mathbf{R}=\left(\mathbf{R}^{1}, \ldots, \mathbf{R}^{n}, \vartheta, b\right)
$$


and introduce the space

$$
H=\left\{\mathbf{R} \mid \varphi_{\alpha}^{i}, \zeta^{i} \in L^{2}(\omega), \alpha=1,2 ; i=1, \ldots, n ; \vartheta, b \in \mathbb{R}\right\}
$$

with norm defined by

$$
\|\mathbf{R}\|_{H}^{2}=\sum_{i=1}^{n} \int_{\omega}\left(m_{i}\left|\zeta^{i}\right|^{2}+I_{\rho_{i}}\left|\varphi^{i}\right|^{2}\right)+\frac{5}{12} M|\vartheta|^{2}+M|b|^{2} .
$$

We further set

$$
V=\left\{\mathbf{R} \in H \mid \varphi_{\alpha}^{i}, \zeta^{i} \in H^{1}(\omega), \alpha=1,2 ; i=1, \ldots, n\right\}
$$

with norm defined by

$$
\|\mathbf{R}\|_{V}^{2}=\sum_{i=1}^{n}\left(\sum_{\alpha}\left\|\varphi_{\alpha}^{i}\right\|_{H^{1}(\omega)}^{2}+\left\|\zeta^{i}\right\|_{H^{1}(\omega)}^{2}\right)+|\vartheta|^{2}+|b|^{2} .
$$

Introduce a continuous seminorm $\sigma(\mathbf{R})$ on $V$ by setting

$$
(\sigma(\mathbf{R}))^{2}=\sum_{i=1}^{n} \int_{\omega}\left\{C_{i}\left[e\left(\varphi^{i}\right)\right]: e\left(\varphi^{i}\right)+G_{i} \varepsilon\left|\varphi^{i}+\nabla \zeta^{i}\right|^{2}\right\} d \omega .
$$

Define a closed subspace $\mathcal{V}$ of $V$ by

$$
\begin{gathered}
\mathbf{R} \in \mathcal{V} \Longleftrightarrow \\
\mathbf{R} \in V,\left.\quad \mathbf{R}^{1}\right|_{\gamma_{1}}=0, \\
\left.\zeta^{i}\right|_{\gamma}=k_{i}^{\prime}[(\cdot-1 / 2) \vartheta+b], \\
\left.\varphi_{1}^{i}\right|_{\gamma}=\left.\varphi_{1}^{j}\right|_{\gamma},\left.\quad \varphi_{2}^{i}\right|_{\gamma}=-k_{i}^{\prime} \vartheta, \quad i, j=1, \ldots, n .
\end{gathered}
$$

Lemma 5. For some $k>0$,

$$
\sigma(\mathbf{R}) \geq k\|\mathbf{R}\|_{V}, \quad \forall \mathbf{R} \in \mathcal{V} .
$$

Proof. It is sufficient to show that

$$
\mathbf{R} \in \mathcal{V}, \quad \sigma(\mathbf{R})=0 \Longrightarrow \mathbf{R}=0
$$

Since $\left.\mathbf{R}^{1}\right|_{\gamma_{1}}=0$ and

$$
\int_{\omega}\left\{C_{i}\left[e\left(\varphi^{i}\right)\right]: e\left(\varphi^{i}\right)+G_{i} \varepsilon\left|\varphi^{i}+\nabla \zeta^{i}\right|^{2}\right\} d \omega=0, \quad i=1, \ldots, n,
$$

it follows from the standard Korn's Lemma that $\mathbf{R}^{1}=0$ in $\omega$. The junction conditions (38) with $i=1$ then show that

$$
\vartheta=b=0,\left.\quad \varphi_{1}^{j}\right|_{\gamma}=0, \quad j=2, \ldots, n .
$$

Use of (38) again with $i=2, \ldots, n$ gives

$$
\left.\varphi_{2}^{i}\right|_{\gamma}=\left.\zeta^{i}\right|_{\gamma}=0, \quad i=2, \ldots, n
$$

Thus $\left.\mathbf{R}^{i}\right|_{\gamma}=0, i=2, \ldots, n$ which, in conjunction with (39) and Korn's Lemma, implies that $\mathbf{R}^{i}=0$ in $\omega, i=2, \ldots, n$. Q.E.D.

In view of the last lemma, $\sigma(\cdot)$ defines a norm on $\mathcal{V}$ equivalent to that induced by the topology of $V$. Let $\mathcal{V}$ be endowed with this norm and let $H$ be identified with its dual space. The usual continuous and dense embeddings 
$\mathcal{V} \subset H \subset \mathcal{V}^{\prime}$ then hold. We denote the pairing between elements $\mathbf{R} \in \mathcal{V}^{\prime}$ and $\hat{\mathbf{R}} \in \mathcal{V}$ by $\langle\mathbf{R}, \hat{\mathbf{R}}\rangle_{\mathcal{V}}$. Consider the variational equation

$$
\begin{aligned}
& \langle\ddot{\mathbf{R}}, \hat{\mathbf{R}}\rangle_{\mathcal{V}}+\sigma(\mathbf{R}, \hat{\mathbf{R}})=\sum_{i=1}^{n} \int_{\omega} f_{3}^{i} \hat{\zeta}^{i} d \omega+\sum_{i=2}^{n} \int_{\omega} \mathbf{M}^{i} \cdot \hat{\varphi}^{i} d \omega \\
& +\sum_{i=2}^{n} k_{i} \int_{\omega}\left\{\left[f_{1}^{i}\left(\xi_{2}-1 / 2\right)-f_{2}^{i} \xi_{1}\right] \hat{\vartheta}+f_{1}^{i} \hat{b}\right\} d \omega, \quad \forall \hat{\mathbf{R}} \in \mathcal{V}, 0<t<T .
\end{aligned}
$$

Let initial data

$$
\mathbf{R}(0)=\mathbf{R}_{0} \in \mathcal{V}, \quad \dot{\mathbf{R}}(0)=\mathbf{R}_{1} \in H
$$

be given. Write

$$
\begin{gathered}
\mathbf{f}^{i}=\sum_{j} f_{j}^{i} \mathbf{a}_{j}^{i}, \quad \mathbf{M}^{i}=\sum_{\alpha} M_{\alpha}^{i} \mathbf{a}_{\alpha}^{i}, \quad i=2, \ldots, n, \\
\mathbf{f}^{1}=f_{3}^{1} \mathbf{a}_{3}^{1}, \quad \mathbf{M}^{1}=0, \quad \mathbf{f}=\left(\mathbf{f}^{1}, \ldots, \mathbf{f}^{n}\right), \quad \mathbf{M}=\left(\mathbf{M}^{1}, \ldots, \mathbf{M}^{n}\right),
\end{gathered}
$$

and suppose that $f_{j}^{i}, M_{\alpha}^{i} \in L^{2}(\omega \times(0, T))$. Then

$$
\begin{aligned}
& \mid \sum_{i=1}^{n} \int_{0}^{T} \int_{\omega}\left(f_{3}^{i} \hat{\zeta}^{i}+\mathbf{M}^{i} \cdot \hat{\varphi}^{i}\right) d \omega d t \\
& \quad+\sum_{i=2}^{n} k_{i} \int_{0}^{T} \int_{\omega}\left\{\left[f_{1}^{i}\left(\xi_{2}-1 / 2\right)-f_{2}^{i} \xi_{1}\right] \hat{\vartheta}+f_{1}^{i} \hat{b}\right\} d \omega d t \mid \\
& \leq C\|\hat{\mathbf{R}}\|_{L^{2}(0, T ; H)}, \quad \forall \hat{\mathbf{R}} \in L^{2}(0, T ; H)
\end{aligned}
$$

for some constant $C$ depending on $\mathbf{f}, \mathbf{M}$. It then follows from standard variational theory that $(40),(41)$ has a unique solution with regularity

$$
\mathbf{R} \in C([0, T] ; \mathcal{V}) \cap C^{1}([0, T] ; H) \cap C^{2}\left([0, T] ; \mathcal{V}^{\prime}\right)
$$

Then the functions $\mathbf{u}^{i}:=\varphi^{i}, W_{3}^{i}:=\zeta^{i}, i=1, \ldots, n$, comprise, by definition, the unique solution of the model consisting of equations of motion (28), boundary conditions (22) and (29), junction conditions (20), (21), (32) (34), and initial conditions (41).

We now wish to pass to the limit in (40), (41) as the shear moduli $G_{i} \rightarrow \infty$. In order to emphasize the dependence of the solution of $(40),(41)$ on these parameters, we denote that solution by $\mathbf{R}^{G}$. The space $\mathcal{V}$ and $\mathcal{V}^{\prime}$ likewise depend on $G$, so we now denote them by $\mathcal{V}_{G}$ and $\mathcal{V}_{G}^{\prime}$, respectively. Let $A_{G}$ denote the Riesz isomorphism of $\mathcal{V}_{G}$ onto $\mathcal{V}_{G}^{\prime}$, so that

$$
\left\langle A_{G} \mathbf{R}, \hat{\mathbf{R}}\right\rangle_{\mathcal{V}_{G}}=\sigma(\mathbf{R}, \hat{\mathbf{R}}), \quad \forall \mathbf{R}, \hat{\mathbf{R}} \in \mathcal{V}_{G}
$$

We define a closed subspace $H_{\infty}$ of $H$ by

$$
H_{\infty}=\left\{\mathbf{R} \in H\left|\zeta^{i} \in H^{1}(\omega), \varphi^{i}=-\nabla \zeta^{i}, i=1, \ldots, n, \zeta^{1}\right|_{\gamma_{1}}=0\right\} .
$$

If $\mathbf{R} \in H_{\infty}$,

$$
\|\mathbf{R}\|_{H_{\infty}}^{2}=\|\mathbf{R}\|_{H}^{2}=\sum_{i=1}^{n} \int_{\omega}\left(m_{i}\left|\zeta^{i}\right|^{2}+I_{\rho_{\imath}}\left|\nabla \zeta^{i}\right|^{2}\right) d \omega+\frac{5}{12} M \vartheta^{2}+M b^{2} .
$$


We further introduce a closed subspace $\mathcal{V}_{\infty}$ of $\mathcal{V}_{G}$ by setting $\mathcal{V}_{\infty}=\mathcal{V}_{G} \cap H_{\infty}$. This space is characterized by

$$
\mathbf{R} \in \mathcal{V}_{\infty} \Longleftrightarrow\left\{\begin{array}{c}
\zeta^{i} \in H^{2}(\omega), \quad \varphi^{i} \in H^{1}(\omega), \quad \varphi^{i}=-\nabla \zeta^{i}, \\
\left.\zeta^{1}\right|_{\gamma_{1}}=0,\left.\quad \nabla \zeta^{1}\right|_{\gamma_{1}}=0, \\
\left.\zeta^{i}\right|_{\gamma}=k_{i}^{\prime}[(\cdot-1 / 2) \vartheta+b],\left.\quad \zeta_{, 1}^{i}\right|_{\gamma}=\left.\zeta_{, 1}^{j}\right|_{\gamma}, \quad i, j=1, \ldots, n .
\end{array}\right.
$$

If $\mathbf{R} \in \mathcal{V}_{\infty}$, we have

$$
\|\mathbf{R}\|_{\mathcal{V}_{\infty}}^{2}=\sum_{i=1}^{n} \int_{\omega} C_{i}\left[e\left(\nabla \zeta^{i}\right)\right]: e\left(\nabla \zeta^{i}\right) d \omega .
$$

Note that $H_{\infty}$ and $\mathcal{V}_{\infty}$ are independent of $G_{i}$. If $H_{\infty}$ is identified with its dual space, we have $\mathcal{V}_{\infty} \subset H_{\infty} \subset \mathcal{V}_{\infty}^{\prime}$ with dense and continuous embeddings. Let $A_{\infty}$ denote the Riesz isomorphism of $\mathcal{V}_{\infty}$ onto $\mathcal{V}_{\infty}^{\prime}$. For $\mathbf{R} \in \mathcal{V}_{\infty}, A_{\infty} \mathbf{R} \in$ $\mathcal{V}_{\infty}^{\prime}$ is the restriction of $A_{G} \mathbf{R}$ to $\mathcal{V}_{\infty}$, that is

$$
\left\langle A_{\infty} \mathbf{R}, \hat{\mathbf{R}}\right\rangle_{\mathcal{V}_{\infty}}=\left\langle A_{G} \mathbf{R}, \hat{\mathbf{R}}\right\rangle_{\mathcal{V}_{G}}, \quad \forall \hat{\mathbf{R}} \in \mathcal{V}_{\infty}
$$

Let $\mathbf{G} \in L^{2}(0, T ; H)$ be defined by

$$
\begin{array}{r}
\sum_{i=1}^{n} \int_{0}^{T} \int_{\omega}\left(f_{3}^{i} \hat{\zeta}^{i}+\mathbf{M}^{i} \cdot \hat{\varphi}^{i}\right) d \omega+\sum_{i=2}^{n} k_{i} \int_{0}^{T} \int_{\omega}\left\{\left[f_{1}^{i}\left(\xi_{2}-1 / 2\right)-f_{2}^{i} \xi_{1}\right] \hat{\vartheta}+f_{1}^{i} \hat{b}\right\} d \omega \\
=(\mathbf{G}, \hat{\mathbf{R}})_{L^{2}(0, T ; H)}, \quad \forall \hat{\mathbf{R}} \in L^{2}(0, T ; H)
\end{array}
$$

Equation (40) may be written

$$
\ddot{\mathbf{R}}+A_{G} \mathbf{R}=\mathbf{G} \text { in } \mathcal{V}_{G}^{\prime}, 0<t<T .
$$

Write

$$
\mathbf{G}=\mathbf{G}_{\infty}+\mathbf{G}_{\infty}^{\perp}, \quad \mathbf{G}_{\infty} \in L^{2}\left(H_{\infty}\right), \mathbf{G}_{\infty}^{\perp} \in L^{2}\left(H_{\infty}^{\perp}\right),
$$

where $H_{\infty}^{\perp}$ denotes the orthogonal complement of $H_{\infty}$ in $H$ and where $L^{2}\left(H_{\infty}\right), L^{2}\left(H_{\infty}^{\perp}\right)$ stand in place of $L^{2}\left(0, T ; H_{\infty}\right), L^{2}\left(0, T ; H_{\infty}^{\perp}\right)$, respectively. We similarly write $C\left(H_{\infty}\right), L^{\infty}(V)$, etc., in place of $C\left([0, T] ; H_{\infty}\right)$, $L^{\infty}(0, T ; V)$, etc. We shall also write $G \rightarrow \infty$ to mean that $G_{i} \rightarrow \infty$ for $i=1, \ldots, n$. The main result of this section is the following theorem.

Theorem 6. Assume that

$$
\mathbf{R}_{0} \in \mathcal{V}_{\infty}, \quad \mathbf{R}_{1} \in H_{\infty}, \quad \mathbf{G} \in L^{2}(0, T ; H) .
$$

There is a function $\mathbf{R} \in C\left(\mathcal{V}_{\infty}\right)$ with $\dot{\mathbf{R}} \in C\left(H_{\infty}\right)$ and $\ddot{\mathbf{R}} \in L^{2}\left(\mathcal{V}_{\infty}^{\prime}\right)$ such that as $G \rightarrow \infty$,

$$
\begin{gathered}
\mathbf{R}^{G} \rightarrow \mathbf{R} \text { weakly in } L^{\infty}(V) \text { and strongly in } L^{\infty}(H) ; \\
\dot{\mathbf{R}}^{G} \rightarrow \dot{\mathbf{R}} \text { weakly in } L^{\infty}(H) \text { and strongly in } L^{\infty}\left(\mathcal{V}_{\infty}^{\prime}\right) ; \\
\ddot{\mathbf{R}}^{G} \rightarrow \ddot{\mathbf{R}} \text { weakly in } L^{2}\left(\mathcal{V}_{\infty}^{\prime}\right) .
\end{gathered}
$$

Moreover, $\mathbf{R}$ is the unique solution of

$$
\begin{gathered}
\ddot{\mathbf{R}}+A_{\infty} \mathbf{R}=\mathbf{G}_{\infty}, \quad 0<t<T \\
\mathbf{R}(0)=\mathbf{R}_{0}, \quad \dot{\mathbf{R}}(0)=\mathbf{R}_{1} .
\end{gathered}
$$


Proof. From (42) we deduce the energy identity

$$
\left\|\dot{\mathbf{R}}^{G}(t)\right\|_{H}^{2}+\left\|\mathbf{R}^{G}(t)\right\|_{\mathcal{V}_{G}}^{2}=\left\|\mathbf{R}_{1}\right\|_{H_{\infty}}^{2}+\left\|\mathbf{R}_{0}\right\|_{\mathcal{V}_{\infty}}^{2}+\int_{0}^{t}\left(\mathbf{G}(s), \dot{\mathbf{R}}^{G}(s)\right)_{H} d s,
$$

from which it follows that

$$
\left\|\dot{\mathbf{R}}^{G}\right\|_{L^{\infty}(H)}^{2}+\left\|\mathbf{R}^{G}\right\|_{L^{\infty}\left(\mathcal{V}_{G}\right)}^{2} \leq C\left(\left\|\mathbf{R}_{1}\right\|_{H_{\infty}}^{2}+\left\|\mathbf{R}_{0}\right\|_{\mathcal{V}_{\infty}}^{2}+\|\mathbf{G}\|_{L^{2}(H)}^{2}\right)
$$

for some constant $C$ independent of $G$. Since the right side of (46) is independent of $G$, as $G \rightarrow \infty$ we obtain

$$
\dot{\mathbf{R}}^{G} \text { is bounded in } L^{\infty}(H) \text {. }
$$

In addition, by using the definition of the $\mathcal{V}_{G}$ norm, we may conclude from (46) that for $\alpha=1,2$ and $i=1, \ldots, n$,

$$
\begin{gathered}
\varphi_{\alpha, \beta}^{i, G}+\varphi_{\beta, \alpha}^{i, G} \text { is bounded in } L^{\infty}\left(L^{2}(\omega)\right), \\
\sqrt{G_{i}}\left|\varphi^{i, G}+\nabla \zeta^{i, G}\right| \text { is bounded in } L^{\infty}\left(L^{2}(\omega)\right) .
\end{gathered}
$$

Our notation is as follows:

$$
\begin{gathered}
\mathbf{R}^{G}=\left(\mathbf{R}^{1, G}, \ldots, \mathbf{R}^{n, G}, \vartheta^{G}, b^{G}\right), \\
\mathbf{R}^{i, G}=\sum_{\alpha} \varphi_{\alpha}^{i, G} \mathbf{a}_{\alpha}^{i}+\zeta^{i, G} \mathbf{a}_{3}^{i}:=\varphi^{i, G}+\zeta^{i, G} \mathbf{a}_{3}^{i} .
\end{gathered}
$$

Since $\left.\mathbf{R}^{1, G}\right|_{\gamma_{1}}=0$, it follows from (48) and Korn's lemma for 2-dimensional linear elasticity that

$$
\varphi_{\alpha}^{1, G} \text { is bounded in } L^{\infty}\left(H^{1}(\omega)\right), \alpha=1,2 .
$$

Then, from (49) and (50) we may conclude that

$$
\zeta_{, \alpha}^{1, G} \text { is bounded in } L^{\infty}\left(L^{2}(\omega)\right), \alpha=1,2 .
$$

Statement (47) implies that $t \mapsto \mathbf{R}^{G}(t):[0, T] \mapsto H$ is equicontinuous in $G>0$. Further, (47) and (43) imply that

$$
\mathbf{R}^{G} \text { is bounded in } L^{\infty}(H),
$$

so, in particular,

$$
\zeta^{1, G} \text { is bounded in } L^{\infty}\left(L^{2}(\omega)\right)
$$

and

$$
\vartheta^{G}, b^{G} \text { are bounded in } L^{\infty}(\mathbb{R}):=L^{\infty}(0, T ; \mathbb{R}) .
$$

It follows from (51) and (52) that

$$
\zeta^{1, G} \text { is bounded in } L^{\infty}\left(H^{1}(\omega)\right) .
$$

We now use (50), (53) and (54), together with the junction conditions (38), to conclude that

$$
\left.\zeta^{i, G}\right|_{\gamma},\left.\varphi_{\alpha}^{i, G}\right|_{\gamma} \text { are bounded in } L^{\infty}\left(H^{1 / 2}(\gamma)\right), \quad i=2, \ldots, n .
$$

Then (48) and Korn's lemma give

$$
\varphi_{\alpha}^{i, G} \text { is bounded in } L^{\infty}\left(H^{1}(\omega)\right), \quad \alpha=1,2 ; i=2, \ldots, n,
$$

and then, as above, we may conclude that

$$
\zeta^{i, G} \text { is bounded in } L^{\infty}\left(H^{1}(\omega)\right), \quad i=2, \ldots, n \text {. }
$$


We may now conclude from (50), (53) - (56), upon passing to the limit as $G \rightarrow \infty$ through an appropriate subnet of $G>0$, that for some $\mathbf{R} \in L^{\infty}(V)$,

$$
\begin{gathered}
\mathbf{R}^{G} \rightarrow \mathbf{R} \text { weakly* in } L^{\infty}(V), \\
\dot{\mathbf{R}}^{G} \rightarrow \dot{\mathbf{R}} \text { weakly* in } L^{\infty}(H), \\
\varphi_{\alpha}^{i, G}+\nabla \zeta_{, \alpha}^{i, G} \rightarrow 0 \text { strongly in } L^{\infty}\left(L^{2}(\omega)\right) .
\end{gathered}
$$

Then, writing $\mathbf{R}=\left(\mathbf{R}^{1}, \ldots, \mathbf{R}^{n}, \vartheta, b\right), \mathbf{R}^{i}=\varphi^{i}+\zeta^{i} \mathbf{a}_{3}^{i}$ as above, we have

$$
\varphi^{i}=-\nabla \zeta^{i}
$$

Therefore $\zeta^{i} \in L^{\infty}\left(H^{2}(\omega)\right), \mathbf{R} \in L^{\infty}\left(\mathcal{V}_{\infty}\right), \dot{\mathbf{R}} \in L^{\infty}\left(H_{\infty}\right)$. Since $\mathbf{R}^{G}$ is uniformly bounded and equicontinuous from $[0, T]$ into $H$, we also have

$$
\mathbf{R}^{G} \rightarrow \mathbf{R} \text { in } C(H) .
$$

In particular, $\mathbf{R}(0)=\mathbf{R}_{0}$.

Let $\hat{\mathbf{R}} \in L^{2}\left(\mathcal{V}_{\infty}\right)$ and take the scalar product in $L^{2}(H)$ of $(42)$ with $\hat{\mathbf{R}}$. One obtains

$$
\int_{0}^{T}\left\langle\ddot{\mathbf{R}}_{\infty}^{G}, \hat{\mathbf{R}}\right\rangle_{\mathcal{V}_{\infty}} d t=\int_{0}^{T}\left(\mathbf{G}_{\infty}, \hat{\mathbf{R}}\right)_{H_{\infty}} d t-\int_{0}^{T}\left(\mathbf{R}^{G}, \hat{\mathbf{R}}\right)_{\mathcal{V}_{G}} d t
$$

where $\ddot{\mathbf{R}}_{\infty}^{G} \in \mathcal{V}_{\infty}^{\prime}$ denotes the restriction of $\ddot{\mathbf{R}}^{G}$ to $\mathcal{V}_{\infty}$. Therefore

$$
\left|\int_{0}^{T}\left\langle\ddot{\mathbf{R}}_{\infty}^{G}, \hat{\mathbf{R}}\right\rangle_{\mathcal{V}_{\infty}} d t\right| \leq C\left(\left\|\mathbf{G}_{\infty}\right\|_{L^{2}\left(H_{\infty}\right)}+\left\|\mathbf{R}^{G}\right\|_{L^{2}\left(\mathcal{V}_{G}\right)}\right)\|\hat{\mathbf{R}}\|_{L^{2}\left(\mathcal{V}_{\infty}\right)}
$$

It follows that

$$
\ddot{\mathbf{R}}_{\infty}^{G} \text { is bounded in } L^{2}\left(\mathcal{V}_{\infty}^{\prime}\right)
$$

Then

$$
\ddot{\mathbf{R}}_{\infty}^{G} \rightarrow \ddot{\mathbf{R}} \quad \text { weakly in } L^{2}\left(\mathcal{V}_{\infty}^{\prime}\right) .
$$

By the Arzela-Ascoli Theorem,

$$
\dot{\mathbf{R}}_{\infty}^{G} \rightarrow \dot{\mathbf{R}} \text { strongly in } L^{2}\left(\mathcal{V}_{\infty}^{\prime}\right)
$$

so that $t \mapsto \dot{\mathbf{R}}(t)$ is strongly continuous into $\mathcal{V}_{\infty}^{\prime}$ and

$$
\dot{\mathbf{R}}(0)=\lim _{G \rightarrow \infty} \dot{\mathbf{R}}_{\infty}^{G}(0)=\mathbf{R}_{1} .
$$

Upon passing to the limit in (57) we obtain

$$
\int_{0}^{T}\left[\langle\ddot{\mathbf{R}}, \hat{\mathbf{R}}\rangle_{\mathcal{V}_{\infty}}+(\mathbf{R}, \hat{\mathbf{R}})_{\mathcal{V}_{\infty}}\right] d t=\int_{0}^{T}\left(\mathbf{G}_{\infty}, \hat{\mathbf{R}}\right)_{H_{\infty}} d t, \quad \forall \hat{\mathbf{R}} \in L^{2}\left(\mathcal{V}_{\infty}\right)
$$

which is just the variational form of (44). On the other hand, the problem (44), (45) has a unique solution with $\mathbf{R} \in C\left(\mathcal{V}_{\infty}\right) \cap C^{1}\left(H_{\infty}\right) \cap C^{2}\left(\mathcal{V}_{\infty}^{\prime}\right)$. Therefore, convergence of $\mathbf{R}^{G}$ to this solution is through the entire net $G>$ 0 . 


\subsection{Interpretation of the model}

Equation (44) is the same as the variational equation

$$
\langle\ddot{\mathbf{R}}, \hat{\mathbf{R}}\rangle_{\mathcal{V}_{\infty}}+(\mathbf{R}, \hat{\mathbf{R}})_{\mathcal{V}_{\infty}}=\left(\mathbf{G}_{\infty}, \hat{\mathbf{R}}\right)_{H_{\infty}}, \quad \forall \hat{\mathbf{R}} \in \mathcal{V}_{\infty}, 0<t<T
$$

The function $\mathbf{R}$ has the form $\left(\mathbf{R}^{1}, \ldots, \mathbf{R}^{n}, \vartheta, b\right)$, where $\mathbf{R}^{i}=-\nabla \zeta^{i}+\zeta^{i} \mathbf{a}_{3}^{i}$. Since $\mathbf{R}$ takes values in $\mathcal{V}_{\infty}$, the components of $\mathbf{R}$ must satisfy the boundary conditions

$$
\zeta^{1}\left(1, \xi_{2}, t\right)=\frac{\partial \zeta^{1}}{\partial \xi_{1}}\left(1, \xi_{2}, t\right)=0, \quad 0<\xi_{2}<1
$$

and the geometric junction conditions

$$
\begin{gathered}
\zeta^{i}\left(0, \xi_{2}, t\right)=k_{i}^{\prime}\left[\left(\xi_{2}-1 / 2\right) \vartheta+b\right], \\
\frac{\partial \zeta^{i}}{\partial \xi_{1}}\left(0, \xi_{2}, t\right)=\frac{\partial \zeta^{j}}{\partial \xi_{1}}\left(0, \xi_{2}, t\right), \quad 0<\xi_{2}<1 .
\end{gathered}
$$

Note that on $\gamma$ and $\gamma_{1}, \partial \zeta^{i} / \partial \xi_{1}$ is the derivative of $\zeta^{i}$ in the direction of the normal vector $\nu$, up to a sign.

One has

$$
\begin{aligned}
\left(\mathbf{G}_{\infty}, \hat{\mathbf{R}}\right)_{H_{\infty}}= & \sum_{i=1}^{n} \int_{\omega}\left(f_{3}^{i} \hat{\zeta}^{i}-\mathbf{M}^{i} \cdot \nabla \hat{\zeta}^{i}\right) d \omega \\
& +\sum_{i=2}^{n} k_{i} \int_{\omega}\left\{\left[f_{1}^{i}\left(\xi_{2}-1 / 2\right)-f_{2}^{i} \xi_{1}\right] \hat{\vartheta}+f_{1}^{i} \hat{b}\right\} d \omega \\
= & \sum_{i=1}^{n}\left\{\int_{\omega}\left(f_{3}^{i}+\operatorname{div} \mathbf{M}^{i}\right) \hat{\zeta}^{i} d \omega-\int_{\partial \omega} \hat{\zeta}^{i}\left(\boldsymbol{\nu}^{i} \cdot \mathbf{M}^{i}\right) d s\right\} \\
& +\sum_{i=2}^{n} k_{i} \int_{\omega}\left\{\left[f_{1}^{i}\left(\xi_{2}-1 / 2\right)-f_{2}^{i} \xi_{1}\right] \hat{\vartheta}+f_{1}^{i} \hat{b}\right\} d \omega
\end{aligned}
$$

where $\mathbf{M}^{1}=0$. Moreover, at least formally,

$$
\begin{aligned}
& \langle\ddot{\mathbf{R}}, \hat{\mathbf{R}}\rangle_{\mathcal{V}_{\infty}}=\sum_{i=1}^{n} \int_{\omega}\left(m_{i} \ddot{\zeta}^{i} \hat{\zeta}^{i}+I_{\rho_{i}} \nabla \ddot{\zeta}^{i} \cdot \nabla \hat{\zeta}^{i}\right) d \omega+M\left(\frac{5}{12} \ddot{\vartheta} \hat{\vartheta}+\ddot{b} \hat{b}\right) \\
& =\sum_{i=1}^{n}\left\{\int_{\omega}\left(m_{i} \ddot{\zeta}^{i}-I_{\rho_{i}} \Delta \ddot{\zeta}^{i}\right) \hat{\zeta}^{i} d \omega+\int_{\partial \omega} I_{\rho_{i}} \hat{\zeta}^{i}\left(\nu^{i} \cdot \nabla \ddot{\zeta}^{i}\right) d s\right\} \\
& +M\left(\frac{5}{12} \ddot{\vartheta} \hat{\vartheta}+\ddot{b} \hat{b}\right) \text {. }
\end{aligned}
$$


Also, from Green's formula (26), we have

$$
\begin{aligned}
(\mathbf{R}, \hat{\mathbf{R}})_{\mathcal{V}_{\infty}} & =\sum_{i=1}^{n} \int_{\omega} C_{i}\left[e\left(\nabla \zeta^{i}\right)\right]: e\left(\nabla \hat{\zeta}^{i}\right) d \omega \\
= & \sum_{i=1}^{n}\left\{\int_{\partial \omega}\left(\nabla \hat{\zeta}^{i}\right) \cdot\left(C_{i}\left[e\left(\nabla \zeta^{i}\right)\right] \boldsymbol{\nu}^{i}\right) d s-\int_{\omega}\left(\nabla \hat{\zeta}^{i}\right) \cdot \operatorname{div} C_{i}\left[e\left(\nabla \zeta^{i}\right)\right] d \omega\right\} \\
= & \sum_{i=1}^{n}\left\{\int_{\partial \omega}\left(\nabla \hat{\zeta}^{i}\right) \cdot\left(C_{i}\left[e\left(\nabla \zeta^{i}\right)\right] \boldsymbol{\nu}^{i}\right) d s-\int_{\partial \omega} \hat{\zeta}^{i} \sum_{\alpha, \beta} C_{i, \alpha}^{\alpha \beta}\left[e\left(\nabla \zeta^{i}\right)\right] \nu_{\beta} d s\right. \\
& \left.+\int_{\omega} \hat{\zeta}^{i} \sum_{\alpha, \beta} C_{i, \alpha \beta}^{\alpha \beta}\left[e\left(\nabla \zeta^{i}\right)\right] d \omega\right\} .
\end{aligned}
$$

It follows from (58) and (61) - (63) that the equation of motion of the $i$ th plate is

$$
m_{i} \ddot{\zeta}^{i}-I_{p_{i}} \Delta \ddot{\zeta}^{i}+\sum_{\alpha, \beta} C_{i, \alpha \beta}^{\alpha \beta}\left[e\left(\nabla \zeta^{i}\right)\right]=f_{3}^{i}+\operatorname{div} \mathbf{M}^{i}, \quad\left(\mathbf{M}^{1}=0\right)
$$

which is a version of the dynamic Kirchhoff plate equation. In addition, one obtains the boundary conditions

$$
\begin{gathered}
\left\{\begin{array}{l}
\sum_{\alpha, \beta} C_{i}^{\alpha \beta}\left[e\left(\nabla \zeta^{i}\right)\right] \nu_{\alpha} \nu_{\beta}=0 \text { on } \partial \omega \backslash \gamma, i=2, \ldots, n, \\
\sum_{\alpha, \beta} C_{1}^{\alpha \beta}\left[e\left(\nabla \zeta^{1}\right)\right] \nu_{\alpha} \nu_{\beta}=0 \text { on } \partial \omega \backslash\left(\gamma \cup \gamma_{1}\right),
\end{array}\right. \\
\left\{\begin{array}{r}
I_{\rho_{i}}\left(\nu^{i} \cdot \nabla \ddot{\zeta}^{i}\right)-\sum_{\alpha, \beta} C_{i, \alpha}^{\alpha \beta}\left[e\left(\nabla \zeta^{i}\right)\right] \nu_{\beta}-\frac{\partial}{\partial \tau} \sum_{\alpha, \beta} C_{i}^{\alpha \beta}\left[e\left(\nabla \zeta^{i}\right)\right] \nu_{\alpha} \tau_{\beta} \\
=-\nu^{i} \cdot \mathbf{M}^{i} \text { on } \partial \omega \backslash \gamma, i=2, \ldots, n, \\
I_{\rho_{1}}\left(\nu^{1} \cdot \nabla \ddot{\zeta}^{1}\right)-\sum_{\alpha, \beta} C_{1, \alpha}^{\alpha \beta}\left[e\left(\nabla \zeta^{1}\right)\right] \nu_{\beta}-\frac{\partial}{\partial \tau} \sum_{\alpha, \beta} C_{1}^{\alpha \beta}\left[e\left(\nabla \zeta^{1}\right)\right] \nu_{\alpha} \tau_{\beta} \\
=0 \text { on } \partial \omega \backslash\left(\gamma \cup \gamma_{1}\right),
\end{array}\right.
\end{gathered}
$$

where $\boldsymbol{\tau}=\left(\tau_{1}, \tau_{2}\right):=\left(-\nu_{2}, \nu_{1}\right)$ and $\partial / \partial \tau=\sum_{\alpha} \tau_{\alpha} \partial / \partial \xi_{\alpha}$. There is also the variational junction condition

$$
\begin{gathered}
\sum_{i=1}^{n} \int_{\gamma}\left(\left\{I_{\rho_{i}} \boldsymbol{\nu}^{i} \cdot \nabla \ddot{\zeta}^{i}-\sum_{\alpha, \beta} C_{i, \alpha}^{\alpha \beta}\left[e\left(\nabla \zeta^{i}\right)\right] \nu_{\beta}\right\} \hat{\zeta}^{i}+\left(\nabla \hat{\zeta}^{i}\right) \cdot\left(C_{i}\left[e\left(\nabla \zeta^{i}\right)\right] \boldsymbol{\nu}^{i}\right)\right) d \xi_{2} \\
+M\left(\frac{5}{12} \ddot{\vartheta} \hat{\vartheta}+\ddot{b} \hat{b}\right)=-\sum_{i=2}^{n} \int_{\gamma} \hat{\zeta}^{i}\left(\boldsymbol{\nu}^{i} \cdot \mathbf{M}^{i}\right) d s \\
+\sum_{i=2}^{n} k_{i} \int_{\omega}\left\{\left[f_{1}^{i}\left(\xi_{2}-1 / 2\right)-f_{2}^{i} \xi_{1}\right] \hat{\vartheta}+f_{1}^{i} \hat{b}\right\} d \omega . \quad(67)
\end{gathered}
$$


On $\gamma$ we have

$$
\left\{\begin{aligned}
& \hat{\zeta}^{i}=k_{i}^{\prime}\left[\left(\xi_{2}-1 / 2\right) \hat{\vartheta}+\hat{b}\right] \\
& \boldsymbol{\nu}^{i} \cdot \mathbf{M}^{i}=-M_{1}^{i}, \\
& I_{\rho_{i}} \nu^{i} \cdot \nabla \ddot{\zeta}^{i}-\sum_{\alpha, \beta} C_{i, \alpha}^{\alpha \beta}\left[e\left(\nabla \zeta^{i}\right)\right] \nu_{\beta}=-I_{\rho_{i}} \ddot{\zeta}_{, 1}^{i}+\sum_{\alpha} C_{i, \alpha}^{1 \alpha}\left[e\left(\nabla \zeta^{i}\right)\right] \\
&\left(\nabla \hat{\zeta}^{i}\right) \cdot\left(C_{i}\left[e\left(\nabla \zeta^{i}\right)\right]\right) \nu^{i}=\sum_{\alpha} C_{i}^{1 \alpha}\left[e\left(\nabla \zeta^{i}\right)\right] \hat{\zeta}_{, \alpha}^{i} \\
&=\hat{\zeta}_{, 1}^{1} C_{i}^{11}\left[e\left(\nabla \zeta^{i}\right)\right]-k_{i}^{\prime} \hat{\vartheta} C_{i}^{12}\left[e\left(\nabla \zeta^{i}\right)\right] .
\end{aligned}\right.
$$

It follows from (67) and (68) that the following mechanical junction conditions must hold:

$$
\begin{gathered}
\sum_{i=1}^{n} C_{i}^{11}\left[e\left(\nabla \zeta^{i}\right)\right]=0, \\
M \ddot{b}=\sum_{i=1}^{n} k_{i}^{\prime} \int_{\gamma}\left(I_{\rho_{i}} \ddot{\zeta}_{, 1}^{i}-\sum_{\alpha} C_{i, \alpha}^{1 \alpha}\left[e\left(\nabla \zeta^{i}\right)\right]+M_{1}^{i}\right) d \xi_{2}+\sum_{i=2}^{n} k_{i} \int_{\omega} f_{1}^{i} d \omega, \\
\frac{5}{12} M \ddot{\vartheta}=\sum_{i=1}^{n} k_{i}^{\prime} \int_{\gamma}\left\{\left(\xi_{2}-1 / 2\right)\left(I_{\rho_{i}} \ddot{\zeta}_{, 1}^{i}-\sum_{\alpha} C_{i, \alpha}^{1 \alpha}\left[e\left(\nabla \zeta^{i}\right)\right]+M_{1}^{i}\right)\right. \\
\left.+C_{i}^{12}\left[e\left(\nabla \zeta^{i}\right)\right]\right\} d \xi_{2}+\sum_{i=2}^{n} k_{i} \int_{\omega}\left[f_{1}^{i}\left(\xi_{2}-1 / 2\right)-f_{2}^{i} \xi_{1}\right] d \omega,
\end{gathered}
$$

where $M_{1}^{1}=0$.

REMARK 7 . When $n=2$ and $\mathcal{P}_{1}$ is orthogonal to $\mathcal{P}_{2}$, the above junction conditions reduce to

$$
\begin{gathered}
C_{1}^{11}\left[e\left(\nabla \zeta^{1}\right)\right]+C_{2}^{11}\left[e\left(\nabla \zeta^{2}\right)\right]=0 \\
\varepsilon \rho_{2} \ddot{b}=k_{1}^{\prime} \int_{\gamma}\left(I_{\rho_{1}} \ddot{\zeta}_{, 1}^{1}-\sum_{\alpha} C_{1, \alpha}^{1 \alpha}\left[e\left(\nabla \zeta^{1}\right)\right]\right) d \xi_{2}+\int_{\omega} f_{1}^{2} d \omega \\
\frac{5}{12} \varepsilon \rho_{2} \ddot{\vartheta}=k_{1}^{\prime} \int_{\gamma}\left\{\left(\xi_{2}-1 / 2\right)\left(I_{\rho_{1}} \ddot{\zeta}_{, 1}^{1}-\sum_{\alpha} C_{1, \alpha}^{1 \alpha}\left[e\left(\nabla \zeta^{1}\right)\right]\right)\right. \\
\left.+C_{1}^{12}\left[e\left(\nabla \zeta^{1}\right)\right]\right\} d \xi_{2}+\int_{\omega}\left[f_{1}^{2}\left(\xi_{2}-1 / 2\right)-f_{2}^{2} \xi_{1}\right] d \omega
\end{gathered}
$$

where $k_{1}^{\prime}= \pm 1$. If the material properties of the two plates are the same and if one ignores the rotational inertia term $\left(I_{\rho_{1}}=0\right)$, these relations together with $(60)$ are the same as the junction conditions found by Le Dret (c.f. [8, p. 156]).

\section{APPENDIX}

Consider a single Reissner-Mindlin plate with reference surface $\mathcal{P}$ and orthonormal basis $\mathbf{a}_{1}, \mathbf{a}_{2}, \mathbf{a}_{3}$ as described in section 1 . Let $\mathbf{A}_{3}$ be the unit 
vector associated with the Reissner-Mindlin hypothesis. The vector rotation angle associated with the deformation of $\mathcal{P}$ is a vector

$$
\mathbf{\Phi}=\sum_{\alpha} \phi_{\alpha} \mathbf{a}_{\alpha}+\psi_{3} \mathbf{a}_{3}
$$

where $\phi_{1}, \phi_{2}, \psi_{3}$ are certain rotation angles which are defined as follows. The vector $\mathbf{a}_{3}$ may be mapped to $\mathbf{A}_{3}$ through successive rotations $\phi_{1}$ around the $\mathbf{a}_{1}$-axis and $\phi_{2}$ around the (rotated) $\mathbf{a}_{2}$-axis. The angle $\phi_{1}$ (resp., $\phi_{2}$ ) measures combined bending and shearing in the $\mathbf{a}_{2} \mathbf{a}_{3}$-plane (resp., in the $\mathbf{a}_{1} \mathbf{a}_{3}$-plane). If we choose the positive direction of rotation to be counterclockwise, these angles are related to the vector $\mathbf{U}=\mathbf{A}_{3}-\mathbf{a}_{3}=\sum_{j} U_{j} \mathbf{a}_{j}$ by

$$
\tan \phi_{1}=-\frac{U_{2}}{1+U_{3}}, \quad \tan \phi_{2}=\frac{U_{1}}{1+U_{3}},
$$

which obviously linearize to

$$
\phi_{1}=-U_{2}, \quad \phi_{2}=U_{1} .
$$

To define $\psi_{3}$, set

$$
\mathbf{t}_{\alpha}=\frac{\mathbf{a}_{\alpha}+\mathbf{W}_{, \alpha}}{\left|\mathbf{a}_{\alpha}+\mathbf{W}_{, \alpha}\right|}, \quad \mathbf{n}=\frac{\mathbf{t}_{1} \times \mathbf{t}_{2}}{\left|\mathbf{t}_{1} \times \mathbf{t}_{2}\right|} .
$$

The vectors $\mathbf{t}_{\alpha}$ are tangent to the deformed reference surface at $\mathbf{P}\left(\xi_{1}, \xi_{2}\right)$ but are not necessarily orthogonal. Introduce $\mathbf{t}_{\alpha}^{\prime}$ such that $\left(\mathbf{t}_{1}^{\prime}, \mathbf{t}_{2}^{\prime}, \mathbf{n}\right)$ is a direct orthonormal system and

$$
\mathbf{t}_{1}^{\prime} \cdot \mathbf{t}_{2}=\mathbf{t}_{2}^{\prime} \cdot \mathbf{t}_{1}, \quad \mathbf{t}_{1}^{\prime} \cdot \mathbf{t}_{1}>0 .
$$

These conditions uniquely determine $\mathbf{t}_{1}^{\prime}, \mathbf{t}_{2}^{\prime}$. Consider the orthogonal transformation mapping $\mathbf{a}_{1}, \mathbf{a}_{2}, \mathbf{a}_{3}$ to $\mathbf{t}_{1}^{\prime}, \mathbf{t}_{2}^{\prime}, \mathbf{n}$, consisting of three successive rotations: a rotation $\psi_{1}$ around the $\mathbf{a}_{1}$-axis followed by a rotation $\psi_{2}$ around the (rotated) $\mathbf{a}_{2}$-axis such that the two combined rotations maps $\mathbf{a}_{3}$ to $\mathbf{n}$, followed by a rotation $\psi_{3}$ around $\mathbf{n}$. The first two rotations map $\mathbf{a}_{1}, \mathbf{a}_{2}$ onto an orthonormal pair $\mathbf{a}_{1}^{\prime}, \mathbf{a}_{2}^{\prime}$ in the tangent plane at $\mathbf{P}\left(\xi_{1}, \xi_{2}\right)$ and $\psi_{3}$ maps $\mathbf{a}_{1}^{\prime}, \mathbf{a}_{2}^{\prime}$ onto $\mathbf{t}_{1}^{\prime}, \mathbf{t}_{2}^{\prime}$. The angle $\psi_{1}$ (resp., $\psi_{2}$ ) measures bending in the $\mathbf{a}_{2} \mathbf{a}_{3}-$ plane (resp., in the $\mathbf{a}_{1} \mathbf{a}_{3}$-plane); the differences $\phi_{\alpha}-\psi_{\alpha}$ are the shear angles. The angle $\psi_{3}$ is related to twisting in the reference plane and satisfies [3, Chap. VII, Sect. 1.5]

$$
\sin ^{2} \psi_{3}=\frac{1}{2}\left[1-\left(\mathbf{a}_{1}^{\prime} \cdot \mathbf{t}_{1}\right)\left(\mathbf{a}_{2}^{\prime} \cdot \mathbf{t}_{2}\right)-\left(\mathbf{a}_{1}^{\prime} \cdot \mathbf{t}_{2}\right)\left(\mathbf{a}_{2}^{\prime} \cdot \mathbf{t}_{1}\right)\right] .
$$

This linearizes to [3, Chap. VII, Sect. 2.4]

$$
\psi_{3}=\frac{1}{2}\left(W_{2,1}-W_{1,2}\right)=\frac{1}{2} \mathbf{a}_{3} \cdot \operatorname{curl} \mathbf{w} .
$$

It follows from (72) and (73) that the linearized vector rotation angle is

$$
\Phi=-\mathbf{U}_{2} \mathbf{a}_{1}+U_{1} \mathbf{a}_{2}+\frac{1}{2}\left(\mathbf{a}_{3} \cdot \operatorname{curl} \mathbf{w}\right) \mathbf{a}_{3} .
$$

In terms of normal and tangential vectors, this is

$$
\boldsymbol{\Phi}=-(\boldsymbol{\tau} \cdot \mathbf{u}) \boldsymbol{\nu}+(\boldsymbol{\nu} \cdot \mathbf{u}) \boldsymbol{\tau}+\frac{1}{2}\left(\tau \cdot \frac{\partial \mathbf{w}}{\partial \nu}-\nu \cdot \frac{\partial \mathbf{w}}{\partial \tau}\right) \mathbf{a}_{3} .
$$




\section{REFERENCES}

[1] Ciarlet, P. G., Mathematical Elasticity, Vol. I: Three-dimensional Elasticity, Studies in Mathematics and its Applications, Vol. 20, North-Holland, Amsterdam, 1988.

[2] Lagnese, J. and Leugering, G., "Modelling of dynamic networks of thin elastic plates," Math. Meth. Appl. Sci., 16 (1993), 379-407.

[3] Lagnese, J., Leugering, G. and Schmidt, E.J.P.G., Modeling, Analysis and Control of Dynamic Elastic Multi-Link Structures, Birkhäuser, Boston, 1994.

[4] Le Dret, H., "Modélisation d'une plaque pliée," C.R. Acad. Sci. Paris, série I, 304 (1987), 571-573.

[5] Le Dret, H., "Modeling of a folded plate," Comput. Mech., 5 (1990), 401-416.

[6] Le Dret, H., "Folded plates revisited," Comput. Mech., 5 (1989), 345-365.

[7] Le Dret, H., "Vibrations of a folded plate," Modélisation Mathématique et Analyse Numérique, 24 (1990), 501-521.

[8] Le Dret, H., Problèmes Variationnels dans les Multi-domaines, Collection RMA, Vol. 19, Masson, Paris, 1991.

Department of Mathematics, Georgetown University, Washington, DC 20057 USA 